\title{
Lower Approximation Reduction in Ordered Information System with Fuzzy Decision
}

\author{
Xiaoyan Zhang, Weihua Xu \\ School of Mathematics and Statistics, Chongqing University of Technology, Chongqing, China \\ E-mail: zhangxyms@gmail.com, chxuwh@gmail.com \\ Received March 28, 2011; Revised June 5, 2011; accepted June 8, 2011
}

\begin{abstract}
Attribute reduction is one of the most important problems in rough set theory. This paper introduces the concept of lower approximation reduction in ordered information systems with fuzzy decision. Moreover, the judgment theorem and discernable matrix are obtained, in which case an approach to attribute reduction in ordered information system with fuzzy decision is constructed. As an application of lower approximation reduction, some examples are applied to examine the validity of works obtained in our works.
\end{abstract}

Keywords: Fuzzy Decision, Lower Approximation Reduction, Ordered Information Systems, Rough Set

\section{Introduction}

Rough set theory, proposed by Pawlak Z. in the early 1980 s [1], is an extension of classical set theory and can be regarded as a soft computing tool to deal with uncertainty or imprecision information. It was well known that this theory is based upon the classification mechanism, from which the classification can be viewed as an equivalence relation and knowledge blocks induced by it be a partition on universe. For this reason, it has been applied widely and successfully in pattern recognition [2], medical diagnosis [3], granular computing [4] and so on.

Attributes reduction, as one important portion of rough set researching, its main idea is not only to delete redundant attributes but also to preserve the invariability of classification ability. In fact, for the reason of noise or information losing, there have many information systems that the relation is not equivalence relation. Therefore, how to deal with this type of information systems has became a very hot topic in rough set theory. Meantime, many experts have studied attribute reduction by extend ing the equivalence relation to consistent relation, similar relation, dominance relation [5-7] and so on. Also, some useful works, take dominance relation based information systems, [8-14] for example, have been done in detail.

At the same time, for decision makers, there may exist one case that the decision objects are not certain or precision but fuzzy. So the fuzzy decision must be taken into consideration. Our work in this paper is to consider the attribute reduction in ordered information systems with fuzzy decision. Firstly, concept of lower approximation consistent set is proposed by comparing decision attribute values. What is more, lower approximation reduction and judgment theorem is introduced, from which one can define the discernable matrix and find that it is an useful approach to attribute reduction in ordered information system with fuzzy decision. As an application of lower approximation reduction, examples are considered to illustrate the validity of some results obtained in our works.

The rest of this paper is organized as follows. Some preliminary concepts required in our work are briefly recalled in Section 2. In Section 3 the lower approximation reduction in ordered information system with fuzzy decision is investigated. The discernable matrix and approach to attribute reduction are defined in Section 4. Section 5 concludes this paper.

\section{Information System with Fuzzy Decision}

In this section, we shall begin our work with some necessary concepts required. For more detailed description of the theory, please refer to references $[15,16]$.

Definition 2.1. An information system is a quaternion $I=(U, A \cup D, F, G)$, where

$U=\left\{u_{1}, u_{2}, \cdots, u_{n}\right\}$ is nonempty finite set of objects;

$A=\left\{a_{1}, a_{2}, \cdots, a_{p}\right\}$ is nonempty finite set of conditional attributes;

$D=\{d\}$ is set of decision attribute.

$F=\left\{f_{k} \mid U \rightarrow V_{k}, k \leq p\right\}$ is relation set of $U$ and $A$, 
and $V_{k}=\left\{f_{k}\left(u_{i}\right) \mid u_{i} \in U\right\}$ is the domain of $a_{k} \in A$.

$G=\left\{d \mid U \rightarrow V_{d}\right\}$ is the relation set of $U$ and $D$, and $V_{d}=\left\{\mathrm{d}\left(u_{i}\right) \mid u_{i} \in U\right\}$ is the domain of $d \in D$.

Information system with fuzzy decision is that the decision value of objects is expressed as fuzzy form, i.e., for any $u_{i} \in U, d\left(u_{i}\right) \in[0,1]$.

Definition 2.2. Let $I=(U, A \cup D, F, G)$ be an information system and $B \subseteq A$, if denote

$$
R_{B}^{\succ}=\left\{\left(u_{i}, u_{j}\right) \in U \times U \mid f_{k}\left(u_{i}\right) \leq f_{k}\left(u_{j}\right), \forall a_{k} \in B\right\},
$$

then $R_{B}^{\succ}$ means a dominance relation on $U$ with respect to $B$, in which case the information system $I=(U, A \cup D, F, G)$ is called an ordered information system and denoted by $I^{\succeq}$.

If an information system is based on dominance relation and the decision value of objects is fuzzy, then the information system is called ordered information system with fuzzy decision. For convenience, the notation $I_{f}^{\succ}$ is used to express ordered information systems with fuzzy decision, from which the dominance class of any $u_{i} \in U$ is denoted as $\left[u_{i}\right]_{B}^{\succ}=\left\{u_{j} \mid\left(u_{i}, u_{j}\right) \in R_{B}^{\succ}\right\}$.

Proposition 2.1. Let $I_{f}^{\succ}$ be an information system and $B \subseteq A$.

1) $\bar{R}_{B}^{\succ}$ is reflexive, transitive, but not symmetric, so it is not an equivalence relation.

2) If $B_{1} \subseteq B_{2} \subseteq A$, then $R_{A}^{\succ} \subseteq R_{B_{2}}^{\succ} \subseteq R_{B_{1}}^{\succ}$.

3) If $B_{1} \subseteq B_{2} \subseteq A$, then $\left[u_{i}\right]_{A}^{\succeq} \subseteq\left[u_{i}\right]_{B_{2}}^{\succeq} \subseteq\left[u_{i}\right]_{B_{1}}^{\succeq}$.

4) If $u_{j} \in\left[u_{i}\right]_{A}^{\succ}$, then $\left[u_{j}\right]_{A}^{\succ} \subseteq\left[u_{i}\right]_{A}^{\succ}$.

Definition 2.3. For an information system $I_{f}^{\succ}$, the lower and lower approximation sets of $D$ with respect to $A$, which are fuzzy sets, are denoted by $A_{D}^{\succ}$ and $A_{D}^{\succ}$, respectively. And their membership functions are defined by

$$
\begin{aligned}
& \underline{\underline{A_{D}}}\left(u_{i}\right)=\min \left\{d\left(u_{j}\right) \mid u_{j} \in\left[u_{i}\right]_{A}^{\succ}\right\}, \\
& \overline{A_{D}^{\succeq}}\left(u_{i}\right)=\max \left\{d\left(u_{j}\right) \mid u_{j} \in\left[u_{i}\right]_{A}^{\succeq}\right\} .
\end{aligned}
$$

Example 2.1. Consider an ordered information system with fuzzy decision in Table 1.

By computing we have that

$$
\begin{array}{ll}
d\left(u_{1}\right)=0.5, & d\left(u_{2}\right)=0.3, \\
d\left(u_{3}\right)=0.7, & d\left(u_{4}\right)=0.9, \\
d\left(u_{5}\right)=0.1, & d\left(u_{6}\right)=0.6,
\end{array}
$$

and

$$
\begin{array}{ll}
{\left[u_{1}\right]_{A}^{\succeq}=\left\{u_{1}, u_{2}, u_{5}, u_{6}\right\},} & {\left[u_{2}\right]_{A}^{\succeq}=\left\{u_{2}, u_{5}, u_{6}\right\},} \\
{\left[u_{3}\right]_{A}^{\succeq}=\left\{u_{2}, u_{3}, u_{4}, u_{5}, u_{6}\right\},} & {\left[u_{4}\right]_{A}^{\succeq}=\left\{u_{4}, u_{6}\right\},} \\
{\left[u_{5}\right]_{A}^{\succ}=\left\{u_{5}\right\},} & {\left[u_{6}\right]_{A}^{\succ}=\left\{u_{6}\right\} .}
\end{array}
$$

Table 1. An ordered IS with fuzzy decision.

\begin{tabular}{ccccc}
\hline & \multicolumn{5}{c}{ Attributes } \\
\cline { 2 - 5 } & $a_{1}$ & $a_{2}$ & $a_{3}$ & $d$ \\
\hline$u_{1}$ & 1 & 2 & 1 & 0.5 \\
$u_{2}$ & 3 & 2 & 2 & 0.3 \\
$u_{3}$ & 1 & 1 & 2 & 0.7 \\
$u_{4}$ & 2 & 1 & 3 & 0.9 \\
$u_{5}$ & 3 & 3 & 2 & 0.1 \\
$u_{6}$ & 3 & 2 & 3 & 0.6 \\
\hline
\end{tabular}

So we have that

$$
\begin{aligned}
& \underline{A_{D}^{\succeq}}=\frac{0.1}{u_{1}}+\frac{0.1}{u_{2}}+\frac{0.1}{u_{3}}+\frac{0.6}{u_{4}}+\frac{0.1}{u_{5}}+\frac{0.6}{u_{6}}, \\
& \overline{A_{D}^{\succeq}}=\frac{0.6}{u_{1}}+\frac{0.6}{u_{2}}+\frac{0.9}{u_{3}}+\frac{0.9}{u_{4}}+\frac{0.1}{u_{5}}+\frac{0.6}{u_{6}} .
\end{aligned}
$$

\section{Lower Approximation Reduction of Ordered Information System with Fuzzy Decision}

Since dominance relation is not the equivalence relation, the approach to attribute reduction in original information systems is not fitted for ordered information system with fuzzy decision. Therefore, the lower approximation consistent set is constructed and lower approximation reduction is defined, from which the judgment theorem is introduced.

Definition 3.1. Let $I_{f}^{\succ}$ be an information system and $B \subseteq A$. If $A_{D}^{\succ}\left(u_{i}\right)=B_{D}^{\succ}\left(u_{i}\right)$ for any $u_{i} \in U$, then $B$ is called lower approximation consistent set of this information system. Moreover, if any proper subset of $B$ is not a lower approximation consistent set, then $B$ is one lower approximation reduction of this information system.

Example 3.1. (Continued from Example 2.1) If take $B=\left\{a_{2}, a_{3}\right\}$, we will have that $\left[u_{i}\right]_{A}^{\succeq}=\left[u_{i}\right]_{B}^{\succeq}$ holds for any $u_{i} \in U$. Hence $A_{D}^{\succ}\left(u_{i}\right)=B_{D}^{\succ}\left(u_{i}\right)$, that is, $\left\{a_{2}, a_{3}\right\}$ is one lower approximation consistent set of this information system. Moreover, if take $C=\left\{a_{1}, a_{3}\right\}$, by computing we have

$$
\begin{aligned}
& {\left[u_{1}\right]_{C}^{\succ}=\left\{u_{1}, u_{2}, u_{3}, u_{4}, u_{5}, u_{6}\right\},} \\
& {\left[u_{2}\right]_{C}^{\succ}=\left\{u_{2}, u_{5}, u_{6}\right\},} \\
& {\left[u_{3}\right]_{C}^{\succ}=\left\{u_{2}, u_{3}, u_{4}, u_{5}, u_{6}\right\},} \\
& {\left[u_{4}\right]_{C}^{\succ}=\left\{u_{4}, u_{6}\right\},} \\
& {\left[u_{5}\right]_{C}^{\succ}=\left\{u_{2}, u_{5}, u_{6}\right\},} \\
& {\left[u_{6}\right]_{C}^{\succ}=\left\{u_{6}\right\},}
\end{aligned}
$$


and

$$
\underline{C_{D}^{\succ}}=\frac{0.1}{u_{1}}+\frac{0.1}{u_{2}}+\frac{0.1}{u_{3}}+\frac{0.6}{u_{4}}+\frac{0.1}{u_{5}}+\frac{0.6}{u_{6}}=\underline{A_{D}^{\succ}} .
$$

Hence, $\left\{a_{1}, a_{3}\right\}$ is also another lower approximation consistent set. Moreover, we can obtain that $\left\{a_{3}\right\}$ is lower approximation consistent sets. Hence we conclude that $\left\{a_{3}\right\}$ is one and only lower approximation reduction of this information system.

In the next, the judgment theorem in ordered information system with fuzzy decision is proposed.

Theorem 3.1. Let $I_{f}^{\succ}$ be an information system and $B \subseteq A . B$ is a lower approximation consistent set if and only if for every $u_{i}, u_{j} \in U$, if $A_{D}^{\succ}\left(u_{i}\right)<A_{D}^{\succ}\left(u_{j}\right)$, then there exist $a_{k} \in B$ such that $f_{k}\left(\overline{u_{i}}\right)<f_{k}\left(\overline{u_{j}}\right)$.

\section{Proof:}

" $\Rightarrow$ ": Suppose that $f_{k}\left(u_{i}\right) \geq f_{k}\left(u_{j}\right)$ for every $a_{k} \in B$ when $A_{D}^{\succeq}\left(u_{i}\right)<A_{D}^{\succ}\left(u_{j}\right)$. So we can obtain $u_{i} \in\left[u_{j}\right]_{B}^{\succeq}$, that is, $\left[u_{i}\right]_{B}^{\succeq} \subseteq\left[u_{j}\right]_{B}^{\succeq}$. By

$$
\underline{B_{D}}\left(u_{i}\right)=\min \left\{d(u) \mid u \in\left[u_{i}\right]_{B}^{\succ}\right\}
$$

and

$$
\underline{B_{D}^{\succ}}\left(u_{j}\right)=\min \left\{d(u) \mid u \in\left[u_{j}\right]_{B}^{\succeq}\right\},
$$

we have that $B_{D}^{\succ}\left(u_{j}\right) \leq \underline{B_{D}}\left(u_{i}\right)$.

Since $B$ is lower approximation consistent set, we have that $\underline{A_{D}^{\succ}}\left(u_{j}\right)=\underline{B_{D}^{\succ}}\left(u_{j}\right)$ and $\underline{A_{D}^{\succ}}\left(u_{i}\right)=\underline{B_{D}^{\succ}}\left(u_{i}\right)$. So we can obtain $\underline{A_{D}}\left(u_{j}\right) \leq \underline{A_{D}}\left(u_{i}\right)$. It is a contradiction.

“ $\Leftarrow$ ”: Suppose $B$ is not lower approximation consistent set, then there must exist one $u_{i_{0}} \in U$ such that $\underline{A_{D}^{\succ}}\left(u_{i_{0}}\right) \neq \underline{B_{D}^{\succ}}\left(u_{i_{0}}\right)$. According to Proposition 2.1, we have that $\underline{B_{D}^{\succ}}\left(u_{i_{0}}\right)<\underline{A_{D}^{\succ}}\left(u_{i_{0}}\right)$

If we take $u_{j_{0}} \in\left[u_{i_{0}}\right]_{B}^{\succeq}$ such that

$$
d\left(u_{j_{0}}\right)=\min \left\{d(u) \mid u \in\left[u_{i_{0}}\right]_{B}^{\succ}\right\}=\underline{B_{D}^{\succ}}\left(u_{i_{0}}\right) .
$$

We have known that $d\left(u_{j_{0}}\right) \geq \min \left\{d(u) \mid u \in\left[u_{j_{0}}\right]_{A}^{\succ}\right\}$ because of $u_{j_{0}} \in\left[u_{j_{0}}\right]_{A}^{\succ}$. From above, we have that $\underline{A_{D}^{\succ}}\left(u_{i_{0}}\right)>\underline{A_{D}}\left(u_{j_{0}}\right)$, thus there exist $a_{k} \in B$ such that $f_{k}\left(u_{i_{0}}\right)>f_{k}\left(u_{j_{0}}\right)$. It is a contradiction with $u_{j_{0}} \in\left[u_{i_{0}}\right]_{B}^{\succeq}$.

The theorem is proved.

\section{Approach to Lower Approximation Reduction}

In theorem 3.1, we proposed an equivalent description of lower approximation consistent set, from which it can be used to estimate whether the attribute set is an lower approximation consistent set or not. In next, discernable matrix is introduced first and reduction approach is constructed immediately.

Definition 4.1. Let $I_{f}^{\succ}$ be an information system and $D_{f}^{\succeq}=\left\{\left(u_{i}, u_{j}\right) \mid \underline{A_{D}}\left(u_{i}\right)<\underline{A_{D}}\left(u_{j}\right)\right\}$. If denote

$$
D_{f}\left(u_{i}, u_{j}\right)=\left\{\begin{array}{lc}
\left\{a_{k} \in A \mid f_{k}\left(u_{i}\right)<f_{k}\left(u_{j}\right)\right\} & \left(u_{i}, u_{j}\right) \in D_{f}^{\succ} \\
\varnothing & \left(u_{i}, u_{j}\right) \notin D_{f}^{\succ}
\end{array},\right.
$$

then $D_{f}$ is called the lower approximation discernable attribute set between $u_{i}$ and $u_{j}$, and

$$
M_{f}=\left(D_{f}\left(u_{i}, u_{j}\right)\right)_{u_{i}, u_{j} \in U}
$$

is the discernable matrix of this information system.

Theorem 4.1. Let $I_{f}^{\succ}$ be an information system and $B \subseteq A, B$ is lower approximation consistent set if and only if $B \cap D_{f}\left(u_{i}, u_{j}\right) \neq \varnothing$ for all $\left(u_{i}, u_{j}\right) \in D_{f}^{\succ}$.

Proof:

" $\Rightarrow$ ": By theorem 3.1 we have that there exist $a_{k} \in B$ s.t. $f_{k}\left(u_{i}\right)<f_{k}\left(u_{j}\right)$ for $\left(u_{i}, u_{j}\right) \in D_{f}^{\succ}$, then $a_{k} \in D_{f}\left(u_{i}, u_{j}\right)$, hence $B \cap D_{f}\left(u_{i}, u_{j}\right) \neq \varnothing$.

$$
\text { “ } \Leftarrow \text { : For }\left(u_{i}, u_{j}\right) \in D_{f}^{\succ} \text {, if } B \cap D_{f}\left(u_{i}, u_{j}\right) \neq \varnothing \text {, }
$$
then there exist $a_{k} \in B$ s.t. $a_{k} \in D_{f}\left(u_{i}, u_{j}\right)$, that is $f_{k}\left(u_{i}\right)<f_{k}\left(u_{j}\right)$. By Theorem 3.1 we have that $B$ is a lower approximation consistent set of $A$.

The theorem is proved.

Definition 4.2. For an information system $I_{f}^{\succ}$ and $B \subseteq A$, the lower approximation discernable formula is denoted as

$$
\begin{aligned}
F_{f} & =\wedge\left\{\vee\left\{a_{k} \mid a_{k} \in D_{f}\left(u_{i}, u_{j}\right)\right\}, u_{i}, u_{j} \in U\right\} \\
& =\wedge\left\{\vee\left\{a_{k} \mid a_{k} \in D_{f}\left(u_{i}, u_{j}\right)\right\}, u_{i}, u_{j} \in D_{f}^{\succ}\right\} .
\end{aligned}
$$

Theorem 4.2. Let $I_{f}^{\succ}$ be an information system and the minimum alternative normal form is defined as $E_{f}=\underset{k=1}{\vee v}\left(B_{f}^{k}\right)$. If take $B_{f}^{k}=\left\{a_{s}, s=1,2, \cdots, q_{k}\right\}$ then $\left\{B_{f}^{k}, k=1,2, \cdots, p\right\}$ is the set with all its elements have the lower approximation reduction form.

Proof: For any $\left(u_{i}, u_{j}\right) \in D_{f}^{\succ}$, by the definition of minimum alternative normal form and theorem 4.1, we have that $B_{f}^{k}$ is lower approximation consistent set. If one element of $B_{f}^{k}$ in $F_{f}=\underset{k=1}{\vee}\left(B_{f}^{k}\right)$ is deleted and from which $B_{f}^{k}$ becomes $B_{f}^{k}(*)$, then there must exist 
Table 2. Discernable matrix of the IS in example 2.1.

\begin{tabular}{lllllll}
\hline & \multicolumn{5}{c}{ Objects } \\
\cline { 2 - 7 } & $u_{1}$ & $u_{2}$ & $u_{3}$ & $u_{4}$ & $u_{5}$ & $u_{6}$ \\
\hline$u_{1}$ & $\varnothing$ & $\varnothing$ & $\varnothing$ & $a_{1} a_{3}$ & $\varnothing$ & $a_{1} a_{3}$ \\
$u_{2}$ & $\varnothing$ & $\varnothing$ & $\varnothing$ & $a_{3}$ & $\varnothing$ & $a_{3}$ \\
$u_{3}$ & $\varnothing$ & $\varnothing$ & $\varnothing$ & $a_{1} a_{3}$ & $\varnothing$ & $a_{1} a_{2} a_{3}$ \\
$u_{4}$ & $\varnothing$ & $\varnothing$ & $\varnothing$ & $\varnothing$ & $\varnothing$ & $\varnothing$ \\
$u_{5}$ & $\varnothing$ & $\varnothing$ & $\varnothing$ & $a_{3}$ & $\varnothing$ & $a_{3}$ \\
$u_{6}$ & $\varnothing$ & $\varnothing$ & $\varnothing$ & $\varnothing$ & $\varnothing$ & $\varnothing$ \\
\hline
\end{tabular}

$\left(u_{i_{0}}, u_{j_{0}}\right) \in D_{f}^{\succ}$ such that $B_{f}^{k}(*) \cap D_{f}\left(u_{i_{0}}, u_{j_{0}}\right)=\varnothing$. So $B_{f}^{k}(*)$ is not a lower approximation consistent set. Hence $B_{f}^{k}$ is a lower approximation reduction. Since $M_{f}$ includes all $D_{f}\left(u_{i}, u_{j}\right)$, there don't exist other lower approximation reductions that have different forms.

Example 4.1. (Continued from Example 2.1 and Example 3.1) By computing we have Table 2.

Hence, $E_{f}=\left(a_{1} \vee a_{3}\right) \wedge\left\{a_{3}\right\} \wedge\left\{a_{1} \vee a_{2} \vee a_{3}\right\}=a_{3}$, that is to say, $\left\{a_{3}\right\}$ is the one and only lower approximation reduction, from which we have that the conclusion acquired in this Example is consistent with the results in Example 3.1.

\section{Conclusions}

Attribute reduction, as one hot research topic, has played an important role in rough set theory. However, most of information systems are based on dominance relations because of various factors. To acquire brief decision rules from inconsistent ordered information systems with fuzzy decision, attribute reductions are needed. The main aim of this paper is to study the problem. In this paper, the lower approximation consistent set is constructed and approach to attribute reduction in ordered information system with fuzzy decision is proposed. Moreover, the judgment theorem of lower approximation consistent set is obtained and some useful works are done in detail.

\section{References}

[1] Z. Pawlak, "Rough Set," International Journal of Computer and Information Sciences, 1982, Vol. 11, No. 5, pp. 341-356. doi:10.1007/BF01001956

[2] R. W. Swiniarski and A. Showron, "Rough Set Method in Feature Selection and Recognition," Pattern Recognition letter, Vol. 24, No. 6, 2003, pp. 833-849. doi:10.1016/S0167-8655(02)00196-4

[3] H. L. Li and M. H. Chen "Induction of Multiple Criteria Optimal Classification Rules for Biological and Medical
Data," Computers in Biology and Medicine, Vol. 38, No. 1, 2008, 42-45. doi:10.1016/j.compbiomed.2007.07.006

[4] G. Y. Wang, Q. H. Zhang and J. Hu, "An Overview of Granular Computing," CAA I Transactions on Intelligent Systems, Vol. 26, No. 2, 2007, pp. 8-26.

[5] S. Greco, B. Matarazzo and R. Slowinski, "Rough Approximation of a Preference Relation by Dominance Relation,” European Journal of Operation Research, Vol. 117, 1999, pp. 63-83. doi:10.1016/S0377-2217(98)00127-1

[6] S. Greco, B. Matarazzo and R. Slowinski, "Rough Sets Theory for Multicriteria Decision Analysis,” European Journal of Operational Research, Vol. 129, No. 1, 2001, pp. 1-47. doi:10.1016/S0377-2217(00)00167-3

[7] K. Dembczynski, R. Pindur and R. Susmaga, "Dominance-Based Rough Set Classifier without Induction of Decision Rules," Electronic Notes in Theoretical Computer Science, Vol. 82, No. 4, 2003, pp. 84-95. doi:10.1016/S1571-0661(04)80708-4

[8] W. H. Xu, X. Y. Zhang and W. X. Zhang, "Lower Approximation Reduction in Inconsistent Informations Based on Dominance Relations,” Computer Engineering and Applications, Vol. 45, No. 16, 2009, pp. 66-68.

[9] W. H. Xu, X. Y. Zhang and W. X. Zhang, "Lower Approximation Reduction in Inconsistent Target Information System Based on Dominance Relations,” Computer Engineering, Vol. 35, No. 18, 2009, pp. 191-193.

[10] W. H. Xu and W. X. Zhang, "Methods for Knowledge Reduction in Inconsistent Ordered Information Systems," Journal of Applied Mathematics \& computing, Vol. 26, No. 1, 2008, pp. 313-323.

[11] W. H. Xu, X. Y. Zhang, J. M. Zhong and W. X. Zhang, "Attribute Reduction in Ordered Information Systems Based on Evidence Theory,” Knowledge and Information Systems, Vol. 25, No. 1, 2010, pp. 169-184. doi:10.1007/s10115-009-0248-5

[12] W. H. Xu, X. Y. Zhang and W. X. Zhang, "Knowledge Granulation, Knowledge Entropy and Knowledge Uncertainty Measurein Ordered Information Systems,” Applied Soft Computing, Vol. 9, 2009, pp. 1244-1251.

[13] W. H. Xu and W. X. Zhang, "Measuring Roughness of Generalized Rough Sets Induced By a Covering," Fuzzy Sets and Systems, Vol. 158, No. 22, 2007, pp. 2443-2455. doi:10.1016/j.fss.2007.03.018

[14] W. H. Xu, M. W. Shao and W. X. Zhang, "Knowledge Reduction Based on Evidence Reasoning Theory in Ordered Information Systems," Lecture Notes in Artificial Intelligence, Vol. 4092, 2006, pp. 535-547. doi: $10.1007 / 11811220 \_45$

[15] W. X. Zhang, W. Z. Wu, J. Y. Liang, et al., "Theory and Method of Rough Sets,” Science Press, Beijing, 2001.

[16] W. X. Zhang, Y. Leung and W. Z. Wu, "Information System and Knowledge Discovery," Science Press, Beijing, 2003. 